\title{
To Feel or Not to Feel: Empathy and Physician Burnout
}

\author{
Kristen $\mathrm{Kim}^{1}$ (iD \\ Received: 22 November 2017 / Accepted: 6 December 2017 / Published online: 18 December 2017 \\ (C) Academic Psychiatry 2017
}

As a third-year medical student exploring the wards for the first time, I have felt a number of emotions. I felt joy when my homeless, human immunodeficiency viruspositive patient got accepted to an assisted living facility where she could receive help with her medication regimen. I felt guilt when I found myself not wanting to check on my patient with fecal incontinence and probable Clostridium difficile infection. I felt admiration when my patient awaiting an amputation cracked jokes about investing in a new hook to replace her hand. And I felt sadness when my first patient died.

She was a 71-year-old woman with asthma who presented with shortness of breath. She claimed her symptoms felt similar to previous asthma attacks and all she wanted was to receive her nebulizer treatments and go home. However, during her workup, she was found to have a new lung mass, then a liver mass, then colorectal cancer. A couple of days after I first met her, my resident and I went to check on her before rounds and found her sitting at the edge of her bed as she took quick, labored breaths. My resident sent me on some errands and moments later, I returned to the patient's room to see the medical intensive care unit director through a small crack in the door. Firmly, the director said, "I think you are going to die... a couple of days... call your family and get them here as soon as possible."

The next morning, the patient coded twice and as soon as our student conference ended, I ran up to check on her. I logged into her chart and read: "Patient pronounced." After some deliberation and with the encouragement of a critical care doctor who assured me that we had done everything possible and that the family would appreciate

Kristen Kim

kristen.kim@ rutgers.edu

1 Rutgers New Jersey Medical School, Newark, NJ, USA the gesture, I walked into the patient's room and expressed my condolences.

As I left the patient's room, I texted my team:

Me: "My patient passed away... I'm so sad."

Resident: "Why are you sad?"

Initially, I was taken aback by the resident's response and I felt a wave of shame and embarrassment wash over me. I began to question whether I was overreacting to the situation. I sat, or more frankly hid, behind a computer at the nurse's station feeling... dumb. Why am I sad? I only knew her for a few days and we never developed great rapport. I'm in a hospital and this is what happens in hospitals. People die. Don't feel.

A little while later, a resident on another team noticed me by the computer. I told him the same thing I texted the team to which he responded, "You can't feel every one." His simple, matter-of-fact response triggered a thought: maybe I was not the one overreacting but the residents were the ones underreacting. After all, in the world outside the hospital, sadness and grief are considered natural, appropriate human reactions to death so, if anything, I was the one responding normally. Then why did my response feel so wrong? Why did the residents respond with such stoicism to our patient's death?

Every time a patient dies, we as healthcare providers have the option to feel or not to feel. Judging from the second resident's response, it seems that some choose against engaging their emotions not because they should not but rather because they believe they can not. In other words, the resident seemed to believe that it is not possible to emotionally invest in each patient death. Perhaps residents assume it is easier and more efficient to cold block their emotions, cross the patient's name off the census, and finish their notes for the day; the overwhelming pressures and demands of the work day do often take precedence over all else. Or perhaps they realize that there is nothing more they can do clinically for a dead patient and thus triage them out of their minds and focus on what they can do for their other patients. Or perhaps they assume that 
their emotional capacity is limited and are afraid of becoming drained too quickly, of burning out.

With $49 \%$ of physicians reporting that they "often or always experience feelings of burn-out," preventing physician burnout is indeed an important concern [1]. It is thus worthwhile to consider the potential long-term consequences of disengaging our emotions in the clinical setting. Humans are often thought to have limited ability to empathize. Therefore, allowing oneself to "feel every one" may quickly lead to emotional exhaustion, which in fact is one of the main components of burnout as it has been classically defined [2]. According to this logic, it is conceivable that the residents' emotionless responses were manifestations of a burnt out state.

However, consider the possibility that the converse is true. A recent review on the literature exploring the relationship between empathy and burnout among healthcare staff suggests that there exists a negative association between the two [3]. While causality could not be established because of the cross-sectional nature of the studies included, there was consistent evidence that as empathy decreased, burnout increased, and vice versa. Of course, these results could suggest that burnout causes a decrease in empathy, but it is also possible that the relationship is bidirectional or in the opposite direction such that less empathy leads to a greater likelihood of burnout. The reaction of the second aforementioned resident suggests that at least some physicians knowingly withhold emotional responses in certain circumstances. However, by neglecting to enter into the suffering of our patients and to experience natural human emotions, we may not be protecting but actually harming our future patients and ourselves.

Preventing burnout requires a multilevel approach that includes both structural and individual interventions. From an administrative standpoint, maintaining reasonable duty hour requirements is a way to combat burnout; on an individual level, mindfulness, stress management, and small group discussions are possible strategies [4]. In the context of coping with a patient's death during the workday, mindfulness can be especially effective. Mindfulness has been shown to lead to self-regulated behavior, positive emotional states, and declines in mood disturbances and stress [5]. Instead of ignoring our emotions, acknowledging them, if even for just a few minutes a day, can go a long way.

My first time experiencing a patient's death taught me many lessons including the role of emotions on the wards. Ironically, the widely held assumption that emotional engagement leads to burnout may in part be contributing to the prevalence of burnout within the medical field today. Recognizing this and taking measures to manage emotions in the clinical setting in a more mindful way may ultimately lead to a healthier and happier workforce.

Acknowledgements I thank Rashi Aggarwal, MD, Department of Psychiatry, Rutgers New Jersey Medical School, for her insight and thoughtful feedback.

Compliance with Ethical Standards

Disclosures The author states that there is no conflict of interest.

\section{References}

1. 2016 survey of America's physicians. The Physicians Foundation. 1. http://www.physiciansfoundation.org/uploads/default/Biennial Physician_Survey_2016.pdf. Accessed 13 Nov 2017.

2. Maslach C, Jackson SE. The measurement of experienced burnout. J Organ Behav. 1981;2(2):99-113. https://doi.org/10.1002/job. 4030020205.

3. Wilkinson H, Whittington R, Perry L, Eames C. Examining the relationship between burnout and empathy in healthcare professionals: a systematic review. Burn Res. 2017;6:18-29. https://doi.org/10. 1016/j.burn.2017.06.003.

4. West CP, Dyrbye LN, Erwin PJ, Shanafelt TD. Interventions to prevent and reduce physician burnout: a systematic review and metaanalysis. Lancet. 2016;388(10057):2272-81. https://doi.org/10. 1016/s0140-6736(16)31279-x.

5. Brown KW, Ryan RM. The benefits of being present: mindfulness and its role in psychological well-being. J Pers Soc Psychol. 2003;84(4):822-48. https://doi.org/10.1037/0022-3514.84.4.822. 